Alma Mater Studiorum - Università di Bologna DEPARTMENT OF ECONOMICS

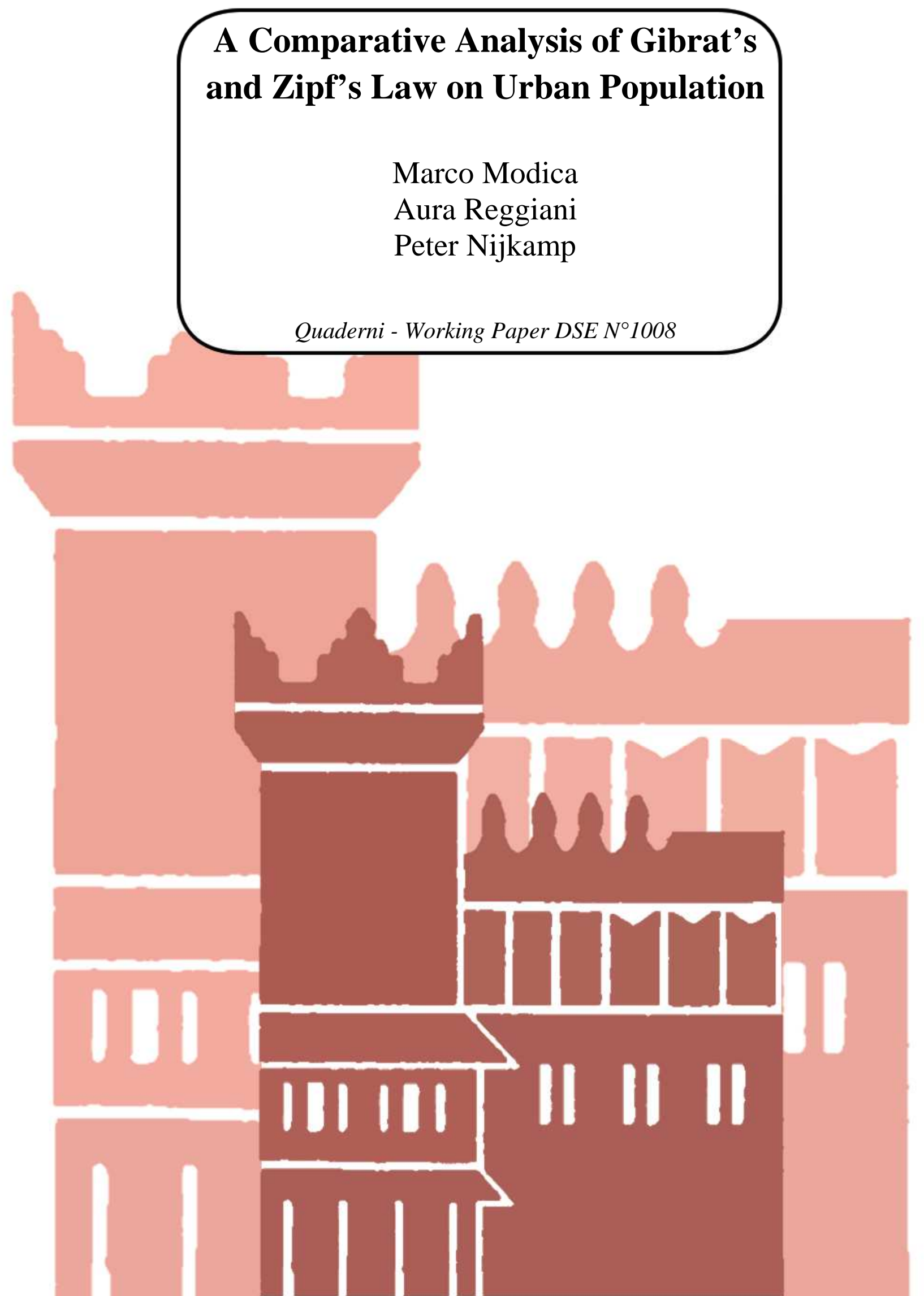




\title{
A Comparative Analysis of Gibrat's and Zipf's Law on Urban Population
}

\author{
Marco Modica* Aura Reggiani** Peter Nijkamp*** \\ *CNR - CERIS Institute for economic research on firms and growth. Via Bassini 15, 20133 Milano, \\ Italy; email: m.modica@ ceris.cnr.it \\ **Department of Economics, University of Bologna, Piazza Scaravilli 2, 40126 Bologna, Italy; email: \\ aura.reggiani@unibo.it \\ ***:Department of Spatial Economics, VU University, De Boelelaan 1105, 1081 HV Amsterdam, The \\ Netherlands; email: p.nijkamp@vu.nl
}

\begin{abstract}
The regional economics and geography literature on urban population size has in recent years shown interesting conceptual and methodological contributions on the validity of Gibrat's Law and Zipf's Law. Despite distinct modeling features, they express similar fundamental characteristics in an equilibrium situation. Zipf's law is formalized in a static form, while its associated dynamic process is articulated by Gibrat's Law. Thus, it is likely that both Zipf's Law and Gibrat's Law share a common root. Unfortunately, empirical investigations on the direct relationship between Gibrat's Law and Zipf's Law are rather rare and not conclusive.

The present paper aims to answer the question whether (a generalisation of) Gibrat's Law allows us to infer Zipf's Law, and vice versa? In our conceptual and applied framework, particular attention will be paid to the role of the mean and the variance of city population as key indicators for assessing the (non-) validity of the generalised Gibrat's Law.

Our empirical experiments are based on a comparative analysis between the dynamics of the urban population of four countries with entirely mutually contrasting spatial-economic and geographic characteristics: Botswana, Germany, Hungary and Luxembourg. We arrive at the following results: if (i) the mean is independent of city size (first necessary condition of Gibrat's law) and (ii) the coefficient of the rank-size rule/Zipf's Law is different from one, then the variance is dependent on city size.
\end{abstract}

Key-words: rank-size rule, Zipf's law, (generalised) Gibrat's Law, hierarchical structure, spatial interaction, city growth.

JEL Classification: C46, D30, O40, R11 


\section{Gibrat's Law vs Zipf's Law: Preliminary Considerations}

Cities all over the world offer an amazing variety in terms of size and growth rates. Despite these differences, systems of cities do not exhibit a random pattern, but a strict regularity in terms of urban hierarchies and inter-urban connectivity. The genesis of such hierarchical perspectives on city size and urban systems can already be found in the seminal contributions of Christaller (1933) and Lösch (1940). The validity of these frameworks has extensively been tested in subsequent statistical experiments in many countries around the world. The conceptual foundation for the existence of central place hierarchies rests on various pillars: agglomeration advantages in cities (depending on city size), smart specialization of industries (depending on scale advantages in different size classes of cities), and transportation and logistics costs (depending on distance frictions between cities or between cities and their hinterlands). Urban hierarchies and inter-urban connectivity are therefore two sides of the same coin (see Paelinck and Nijkamp 1976).

Clearly, it ought to be added that the spatial range of interurban linkages has extended drastically over recent decades. Whereas a century ago, most cities were at best part of an interlinked regional or national system, nowadays cities are often part of a globally connected network.

Surprisingly, despite the complex evolution of current socio-economic spatial networks, two robust empirical regularities seem to hold: Gibrat's law affirming that city growth does not depend on size, and Zipf's law stating the proportionality of a given city size to its rank. ${ }^{1}$

More in details, in 1931, Gibrat observed that the growth rate of a city's population does not depend on the size of the city. In other words, although cities can grow at different rates, no systematic behaviour exists between their growth and their size, so that, according to Gibrat (1931), we cannot affirm that larger cities grow faster than smaller ones or vice versa.

Analytically, we can write the following logarithmic expression, as in Steindl (1968):

$$
\log P(t)=\log P(0)+\varepsilon(1)+\varepsilon(2)+\ldots+\varepsilon(t)
$$

where $P(t)$ is the size of a certain city at time $t, P(0)$ is the initial population, and $\varepsilon(t)$ is a random variable (indicating random shocks), i.i.d random variable with mean $\mu$ and variance $\sigma^{2}$. Equation (1) identifies the logarithm of the size of a given city as the sum of the initial size and past growth rates.

1 Another way to refer to Zipf's Law is a Pareto distribution, with a shape parameter equal to 1 . It is investigated using the so-called rank-size rule. We note here that the slope coefficient of the rank-size rule represents the inverse form of the parameter of the conventional Pareto distribution. For more details, we refer inter alia to Adamic (2000) and Parr (1985). In this paper we refer to Zipf's law (Zipf's distribution), when the rank-size coefficient is exactly equal to 1 . In all the other cases we refer to the rank-size rule (rank-size distribution). 
This law can now be interpreted as follows: "A variate subject to a process of change is said to obey the law of proportionate effect if the change in the variate at any step of the process is a random proportion of the previous value of the variate" (Chesher, 1979, p. 403). The implication of Gibrat's law is that the growth processes of cities have "a common mean (equal to the mean city growth rate) and a common variance" (Gabaix, 1999, p. 741), that is, both the mean and variance have to be independent from the size of the cities.

The second well-known spatial regularity is given by the so-called Zipf's law (on the basis of a first study by Auerbach ${ }^{2}$ in 1913). In 1949, Zipf observed and established that the sizes of the cities in a country are proportional to their rank. This means that in Botswana, for example, the size of largest city, Gaborone, is roughly twice the size of Francistown, the second largest city, three times the third largest city, Molopolole, and so on. Formally, this can be written as:

$$
P_{i}=K R_{i}^{-q}
$$

Equation (2) is known as the rank-size rule and is usually expressed in logarithmic form, as follows:

$$
\log (P)_{i}=\log (K)-q \log \left(R_{i}\right)
$$

where $P_{i}$ is the population of city $i, R_{i}$ is the rank of the $i$ th-city and $K$ is a constant. Zipf's law holds precisely, when the coefficient $q$ is equal to one.

Several interpretations of the Zipf coefficient, $q$, have been proposed in the literature. In principle, the $q$-coefficient can be seen as an indicator of the hierarchical degree of a system of cities (Singer, 1930). In fact, the $q$-coefficient measures how unequal the city distribution is: the higher the $q$-coefficient, the more unequally distributed is the city system. On the contrary, the smaller the value of $q$, the more even is the system of cities (in the extreme, when $q=0$, we have a very even system of cities all of the same size; when $q=\infty$, instead, we have only one city hosting the entire population).

In summary, Gibrat's law expresses the growth process of a certain variable (firm, city, income, wealth, etc.), independent of its size, while Zipf's law presents the static relationship of the size of this variable with its rank. In the field of spatial economics, these two regularities have given rise, especially since the late '90s, to an increasing number of empirical studies, testing cities and economic growth at

2 "The population of a city is inversely proportional to the number indicating its rank among the cities of a given country" (Auerbach, 1915, p. 384). 
various spatial levels (national, regional, local), by means of Gibrat's law and Zipf's law.

It seems that in the majority of urban studies, Zipf's law and Gibrat's law are generally confirmed by empirical data (Eeckhout, 2004; Gonzalez-Val, 2010; Ioannides and Overman, 2003; Gabaix and Ioannides, 2004; Giesen and Suedekum, 2011). However, other studies seem to reject these two empirical regularities (Black and Henderson, 2003; Cuberes, 2011; Gonzalez-Val et al., 2012; Henderson and Wang, 2005). These contrasting results have prompted a continuous debate in the literature, on the (non)validity of Gibrat's law and/or Zipf's law.

These two laws are often theoretically treated together, given their possible complementarily. Indeed, Champernowne (1953) and Simon (1955) have shown that rank-size distributions arise naturally, if Gibrat's law is satisfied. Gabaix (1999) has demonstrated that Gibrat's law leads to a Zipf distribution, while Cordoba (2003) argues that a weak version of Gibrat's law leads to more general rank-size distributions, where weak means that only the mean of the city growth is independent from city size, while its variance can change according to size (Cordoba, 2003). In this setting, Cordoba, for the first time, shows an unknown relationship between the two laws; indeed, he shows that Zipf's law might imply Gibrat's law. Moreover, this setting is related to Garmestani et al. (2007), that have proposed a model that lead to different growth processes for clustering of cities that are aggregated according to similarity of their sizes.

More in details, Cordoba (2008, p. 1463) proposes a: "generalisation of Gibrat's law that allows size to affect the variance of the growth process but not its mean". In particular, one of the implications of Cordoba's generalised model is that non-proportionality of the variance is required to take into account a $q$-coefficient different from one (in Eq. (3)). More specifically, the larger the $q$-coefficient, the more unequal is the distribution, and this makes a growth process more volatile. ${ }^{3}$ On the basis of Cordoba's results, we can outline the following relationships between Zipf's law and Gibrat's law:

(a) If $q=1$, Zipf's law holds. In order that Gibrat's law applies, neither the mean nor the variance of growth can depend on size.

(b) If $q>1$, the distribution is more unequal. In order that Gibrat's law applies, it is necessary that the mean is independent of the city size, but not the variance; indeed, the associated growth process requires that smaller cities face a greater volatility of growth than larger cities.

(c) If $q<1$, the distribution is more evenly distributed. Again, in order that Gibrat's law applies, it is necessary that the mean is independent of the city size, but not the variance. Here, the associated growth process requires that larger cities face a greater volatility of growth than

3 The volatility is a measure of fluctuation of a process. We will use the variance as an indicator of the volatility of an underlying proportionate growth process. 
smaller cities.

It seems, therefore, plausible that Zipf's law and Gibrat's law show features of close kinship, in the sense that they show a bidirectional predictive frame that is able to reveal distinct modeling outcomes, even though in equilibrium it expresses identical fundamental characteristics. A test of this proposition calls for evidence-based research.

Starting from these considerations, the present paper aims to answer the following research question: can (a generalisation of) Gibrat's law allow us to infer Zipf's law and vice versa, by empirically analysing the link between these two laws, in the context of urban growth, and, in particular, the dynamics of city size distributions? In this framework, particular attention will be paid to the role of the mean and variance of the city population as a key indicator for assessing the validity (or non-validity) of the generalised Gibrat's law.

Starting from these considerations, the main challenge and aim of this paper is to empirically explore the above mentioned relationship "Gibrat's law vs Zipf's law", according to the three statements (a)-(c) above. It should be noted that empirical investigations of the relationship "Gibrat's law vs Zipf's law" are still rare in the sense that typically scholars investigate about the validity or non-validity of the laws singularly without any empirical comparison between the estimated coefficients of the two. Here, instead, we want to compare directly the implications of the validity and non-validity of one law to the other in a bidirectional way.

Consistently with Eeckhout (2004), we focus our empirical investigation on the entire city size distributions of four countries (Botswana, Germany, Hungary and Luxembourg) and not only on the upper tail, ${ }^{4}$ as other studies have done (see among others, Giesen and Suedekum, 2011; Guerin-Pace, 1995; Rosen and Resnick, 1980 and Soo, 2005, 2007). Notice that we focus on those particular countries because of their heterogeneity in terms of socio-economic and spatial characteristics.

Our results find evidence of the existence of Gibrat's law for two out of the four preselected countries; we then test the empirical relationship between the (generalised) Gibrat's law and Zipf's law, by considering the dynamics of the hierarchical structure of the various city systems, on the basis of the mean and variance indicators.

The paper is then organised as follows. Section 2 describes the rationale underlying the selection of the four countries under analysis, by focusing on their different spatial economic characteristics and related statistics, while subsequent sections illustrate the results of the empirical analysis devoted to testing

4 Eeckhout (2004) shows that if the city growth does not depend on city size "then the estimated OLS coefficient of the socalled rank-size rule varies depending on the truncation city size, i.e. the inclusion of smaller (larger) cities in the sample, leads to a smaller (larger) coefficient" (Fazio and Modica, 2012, p. 3). 
Gibrat's law (Section 3), as well as the link between Gibrat's law and Zipf's law (Section 4). The paper concludes with some methodological considerations and directions for future research (Section 5).

\section{Choice of Case Studies: Descriptive Analysis and Statistics}

We have selected in our empirical study four distinct countries characterised by different socioeconomic typologies: Botswana, Germany, Hungary and Luxembourg. The selection of these four countries, although mainly illustratively, may be representative of countries with different characteristics; the selection has been made according to size, population density and GDP per capita, according to the representation in Table 1.

Moreover, in Table 2 we report, for each country, some economic indicators (such a GDP per capita, growth rate and percentage of investment over GDP), as well as some other important indicators for the mobility and transportation system (such as the length of railways and roadways, and the number of cars per thousand people). We collected data from the National Institute of Statistics for all four of these countries. ${ }^{5}$ In particular, we collected data from the Central Statistics Office of Botswana, the Institute for Employment Research ${ }^{6}$ (IAB) in Germany, the Hungarian Central Statistical Office and the STATEC-Institute National de la Statistique et des Etudes Economique of Luxembourg

Some points are worth noting here. Botswana is the only non-OECD country, while all the others are OECD countries. Botswana shows the features of a non-advanced ${ }^{7}$ economy; however, it exhibits a trend towards an increase in population and economic growth. Germany was a founding member of the European Community in 1957 (which became the European Union (EU) in 1993); it is central in Europe and is a large country in terms of surface area and population, with an advanced economy. Hungary joined the EU in 2004; it is located in central Europe, but shows a non-advanced economy and a decreasing population. Luxembourg, like Germany, was a founding member of the European Community in 1957; it is a small country, but very central in Europe with a high income per capita. Clearly, other choices could have been made, but the present set of countries aims to represent a sufficiently interesting collection of cases for in-depth investigation.

5 For all countries we have data over all cities from the biggest to the smallest one.

6 The authors wish to thank Uwe Blien and Anette Haas (IAB, Germany), for kindly providing the data used in our study on German cities (Sections 3 and 4).

7 According to the IMF classification. 


\section{< Table 1. About Here > \\ < Table 2. About Here >}

It should be noted that an extensive debate concerns the type of spatial unit under analysis: several studies have been carried out using metropolitan areas, i.e. by considering the entire population in a given city, as well as all populations of suburban areas. Nevertheless, our object is to carry out comparative analysis between the four countries, by also including all the cities in a given country. For these reasons, we consider in our analysis the entities legally defined as cities or villages in their countries, although we are aware that the administrative definition given by legal borders might not fulfill our scopes exactly. In order to have a comparable unit, in all countries we have selected those localities which are similar to a municipality. ${ }^{8}$

Another concern is due to the fact that we have different temporal horizons, which, sometimes, are short. This is the case for Botswana, where we have only two census observations (2000 and 2010), as well as for Hungary, where, although the time span is 30 years (1980-2011), we have only four census observations. For Germany, however, although the time span is 15 years, we have annual data (19932007), so we can conduct a more precise analysis. Finally, Luxembourg have a long time series considering all census data from 1821 until 2011.

Following on from the above observations, in Section 3 we focus our attention on the validity of Gibrat's law, in order to design an analytical framework that is useful for meeting the ultimate goal of our analysis: a comparison of Gibrat's' law and Zipf's law.

\section{Testing Gibrat's' Law: Method and Results}

In this section we will use an OLS regression model and report the results from the parametric analysis. We check dynamic deviations from the proportionality of mean growth and variance to size, by using a method firstly proposed by Kalecki (1945) and subsequently utilised, among others, by Bottazzi et al. (2001). In particular, the adopted model is the following OLS model:

$$
g_{t}^{i}=\beta_{t} g_{t-1}^{i}+\varepsilon_{t}^{i}
$$

8 We encountered some difficultly in making the right choice for Botswana where we also had data for small localities but we chose to collect all localities with ID code 100 , namely villages and cities. 
where $g_{i}(\mathrm{t})$ is the deviation of the logarithm of the population of city $i$ from the mean of the logarithms of the city populations at time $t$, and $\varepsilon$ is the error term. $\beta$ is the parameter to be estimated and "provides an estimate of the divergence/convergence of the size distribution toward its mean" (Bottazzi et al., 2001, p. 1184). Gibrat's law holds if $\beta$ is equal to one. ${ }^{9}$ When $\beta$ is lower than one, this means that size converges towards its mean, namely, the larger a city, the smaller the expected growth. On the contrary, when $\beta$ is greater than one, the larger a city and the larger the expected growth. We test the model for each time-step.

As an indicator of the volatility of the growth process, we use two different measures: i) the variance of growth, $\sigma_{t}^{2}$ ii) the variance ratio, $\theta_{t}$, between the variance of $g$ at time $t, \sigma\left(g_{t}\right)$ and the variance of $g$ at time $t-1, \sigma\left(g_{t-1}\right)$; The reason why we report both the indicators is because Gibrat's law is a dynamic concept, while Zipf's law is static. Including both the measures allow us to be more confident in taking into consideration both the aspects of dynamicity and staticity. Moreover, the variance ratio is also adopted for testing mean reversion and stationarity of the series so it is a further control for Gibrat's law. Formally, it is given by:

$$
\theta_{t}=\frac{\sigma^{2}\left(g_{t}^{i}\right)}{\sigma^{2}\left(g_{t-1}^{i}\right)}
$$

Tables $4 \mathrm{a}$ and $4 \mathrm{~b}$ show the results. Two main conclusions arise from here. Firstly, looking at the parameter $\beta$, Gibrat's law does not always hold (over time). Germany and Luxembourg are clear examples of this intermittency: we can see periods where Gibrat's law holds and others where it does not. Secondly, it seems that the effect of the (non)validity of the law is lengthy: namely, Gibrat's law in general holds (or does not hold) continuously for two or three time windows. Consequently, the first result of our analysis is that testing Gibrat's law requires a data-set of considerable length, or as many possible observations as we can. It is also interesting to note that in general, when Gibrat's law does not apply, the variance at time $t$ is higher than the variance at the previous times; this is denoted by the parameter $\theta$ in Eq. (5) greater than one. This is, of course, consistent with the idea of Gabaix (1999) which, according to Gibrat's law, both the mean and variance of the growth rate have to be independent with respect to the size.

9 We report here only the condition on the estimated $\beta$. However it should be noted that another condition is necessary to affirm that Gibrat's law is in operation, indeed the error terms have to be serially uncorrelated. We, then, add one more lag in Eq. (4) to very this additional condition. In most of the cases the error terms result serially uncorrelated. 
In more detail, by observing $\beta$ in Tables $4 \mathrm{a}$ and $4 \mathrm{~b}$, we can see that in Botswana and Luxembourg, Gibrat's law holds quite often $(\beta=1)$. In Germany it does not apply more than half of the time and in Hungary Gibrat's law never holds.

\author{
< Table 4a. About Here > \\ < Table 4b. About Here >
}

\title{
4. Gibrat's Law and Zipf's Law: A Comparative Study
}

\subsection{Role of the Adopted Parameters}

In the previous sections we have shown that Botswana and Luxembourg seem to obey Gibrat's law, while this seems not to be the case for Germany and Hungary. The final step in our analysis is then the investigation of the relationship between Gibrat's law and the rank-size/Zipf's law, by means of the rules (a), (b) and (c) (outlined in Section 1).

From the operational viewpoint, we investigate the relationship between the $q$-coefficient in Eq. (3) and the estimated parameters $\beta$ and $\theta$ from Eqs. (4)-(5) and $\sigma_{t}^{2}$, on the basis of Cordoba's propositions (a); (b) and (c). In particular, we estimate the $q$-coefficients in the rank-size rule (3) by means of a modification proposed by Gabaix and Ibragimov (2011), according the following:

$$
\log \left(P_{i}\right)=\log (K)-q \log \left(R_{i}-0.5\right)
$$

where $P_{i}, K, q$ and $R_{i}$ are the same as in Eq. (3).

In Tables $5 \mathrm{a}$ and $5 \mathrm{~b}$ we report the estimated $q$-coefficients and the parameters $\beta, \theta$ and $\sigma_{t}^{2}$, according to Eqs. (7), (4) and (5) respectively, for each of the four countries. Overall, by means of these four parameters, we can experiment with the propositions (a), (b) and (c) in Section 1.

Concerning the coefficient $q$ (Eq. (7)), it should be noted that we interpret it as a measure of hierarchy of city size distribution. In this sense a positive change in the estimated $q$-coefficient denotes a situation where larger cities have grown more than smaller ones (in relative terms); thus an increasing $q$ coefficient (see Eq. (7)) reflects the tendency towards agglomeration economies in the country at hand (see Section 1).

For example, an increasing/decreasing $q$-coefficient - indicating changes in the growth rate between large and small cities - should lead to a generalised Gibrat's law. It appears then that the $q$-coefficients, together with the $\beta$-, $\theta$ - and $\sigma_{t}^{2}$-parameters, offer insights into different aspects of the same growth 
process: the $q$-coefficient captures the output of the growth process, while the $\beta$-, $\theta$ - and $\sigma_{t}^{2}$ parameters take into account the mean and variance of the growth process.

In the latter context (regarding the role of the mean and variance), it seems worthwhile to test the different dynamics of the large cities vs the small cities, in order to explore in more detail where a greater volatility shows up. We can then split, for each country, our sample into two halves by defining two sub-samples; one for the large cities and the other one for the smaller cities. We then estimate the parameters $\beta$ and $\theta$ according to Eqs. (4)-(5) and $\sigma_{t}^{2}$ for these two sub-samples. In this way we can analyse, firstly, whether Gibrat's law holds separately for large and small cities; and, secondly, whether the growth process is more volatile even in the upper tail.

\section{< Table 5a. About Here > \\ < Table 5b. About Here >}

In the next Sub-sections, the role of the various parameters $q, \beta, \theta$ and $\sigma_{t}^{2}$ in capturing the relationship "rank-size rule vs Gibrat's law" will be illustrated with reference to the empirical analyses in each of the four countries.

\subsection{Botswana}

Starting with Botswana, we can see that the estimated $q$-coefficient is greater than one for both the years 2001 and 2011, indicating a predominance of larger cities. In particular, in 2011 the estimated $q$ coefficient is slightly greater than that one in 2001, thus showing a tendency - in the last decade towards a higher economic development. By considering the relationship with Gibrat's law, we then investigate condition b) of Section 1. Considering the entire sample, we have already shown that Gibrat's law holds in 2011 with an estimated $\beta$-parameter not significantly different from one $\left(\beta=0.995^{* *}\right) .{ }^{10}$ However, considering the two sub-samples, we find evidence of Gibrat's law for large cities $\left(\beta_{B I G}=0.979 * *\right)$ but not for small ones $\left(\beta_{\text {small }}=0.761\right)$. This indicates that larger cities of the subsample of small cities (i.e. medium size cities) have an expected growth lower than smaller ones. Thus, large and small cities face two different underlying growth processes; however, this is still consistent with proposition $b$ ) of Section 1 predicting that the associated growth process requires that smaller cities face a greater volatility of growth than larger cities. For this reason we now analyse the behaviour

10 Where $* *$ indicates a significance level at $5 \%$. 
of the variance.

The variance ratio for the entire sample in Botswana is greater than one $(\theta=1.078)$, indicating a greater volatility of the process in 2011. This latter condition is not enough to investigate our statements b) of Section 1, because it only refers to the "temporal" non-stability of the variance, without considering the "spatial aspect", namely the (non)independence of the variance with respect to the size of the cities. ${ }^{11}$ For this reason, we analyse the two sub-samples separately, as previously anticipated and we look both at the variance ratio, $\theta$, and variance of growth, $\sigma_{t}^{2}$.

The variance ratio, $\theta$, for large cities shows a striking stability $\left(\theta_{B I G}=1\right)$, while, for the small cities, it is slightly greater than one $\left(\theta_{\text {small }}=1.011\right)$, implying an (increasing) change in the underlying volatility of the growth process for small cities. Moreover, the variance of growth $\sigma_{B I G}^{2}$ is less than $\sigma^{2}$ small.

Given these facts, we can affirm that at time $t$ (2011), the variance is unchanged for large cities but increases for the small ones, indicating a dependence of variance with respect to size; in particular, smaller cities face a greater volatility than large cities.

In summary, statement b) (Section 1), which affirms: "if $q>1$, in order that Gibrat's law occurs, it is necessary that the mean is independent from the city size but not the variance, indeed the associate growth process requires that smaller cities face a greater volatility of growth than larger cities", is satisfied for the whole sample.

\subsection{Germany}

Germany shows an U-shaped $q$-coefficient: it decreases until 1999 and then it increases. In fact Germany shows a lower degree of agglomeration between 1993 and 1999, namely larger cities become less "heavy" in the city system. After 1999, Germany shows again a process of concentration indicated by the increasing $q$-coefficient. By considering the relationship with Gibrat's law, we then investigate condition b) of Section 1.

Considering the entire sample, we have five years in which Gibrat's law holds. In particular, in 1995, 1996, 1999, 2000 and 2003, the estimated $\beta$-parameters are not significantly different from one.

Now if we focus on the period 1993-1999, where a decreasing $q$-coefficient applies, we can note that $\beta_{B I G}$ is significantly lower than one, whereas $\beta_{\text {small }}$ is not significantly different from one in most cases, indicating a situation in which the larger the city, the lower the expected growth. On the contrary, in the

11 It suggests a change in the variance over the time and then this might also imply changes in the dependence of the variance over the size. 
period 2000-2007, ${ }^{12}$ where an increasing $q$-coefficient applies, we can notice that $\beta_{B I G}$ are often not significantly different from one, whereas $\beta_{\text {small }}$ are (most of the time) significantly greater than one, indicating a situation in which the larger the city, the larger the expected growth. In this situation we can figure out the following growth processes: when $q$-coefficient is decreasing, we have modifications on the growth process of large cities; in particular, the larger the city, the lower the expected growth. On the other hand, when $q$ is increasing, small cities present a different growth process, namely the larger the city, the larger the expected growth. However, note that both cases should lead to the same effect on the underlying growth process (i.e. a greater volatility of the variance for small cities), in order to satisfy condition b). For this reason, we analyse the variance ratio, $\theta$ and the variance of growth, $\sigma_{t}^{2}$.

By considering the entire sample, the variance ratio, $\theta$, is often close to unity, but slightly lower than one until 1999 when it becomes stable (and equal to one). Again, this latter condition says few things about the independence of the variance from the size. We then analyse the two sub-samples separately and in particular we analyse those years where Gibrat's law holds (according to proposition b) of Section 1). Unfortunately, we do not have enough observations to make any inference about proposition b) in the period 2000-2007 because Gibrat's law holds only in 2003.

Focusing on the period 1993-1999, it should be noted, firstly, that when Gibrat's law holds, the variance ratios for large cities, $\theta_{B I G}$, are less than one, that is the variance at time $t$ is lower than that at time $t$ - 1 . Instead, the variance ratios for small cities, $\theta_{\text {small }}$, are greater than one. At (any) time $t$, large cities face a lower volatility while small cities face a greater (or almost stable) volatility. Second, the

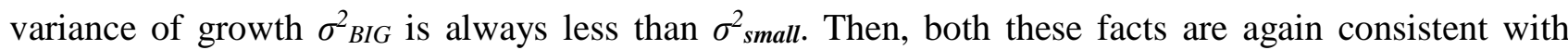
proposition $\mathrm{b}$ ) of Section 1. Again we can affirm that for those years in which Gibrat's law holds $(\beta=1)$, statement b) (Section 1) is satisfied for the whole sample.

\subsection{Hungary}

In Section 3, we have shown that Gibrat's law does not hold in Hungary. The evolution of the $q$ coefficient in this country reflects the tendency to agglomeration in the large cities: indeed the estimated $q$-coefficient is increasing and greater than one. In this situation we can check for statement b) of Section 1. Unfortunately Gibrat's law never holds, since the $\beta$-coefficients are always

12 Notice that in the years in which the $q$-coefficient is equal to that of previous year (i.e. 1998, 1999 and 2000), Gibrat's law holds. The stability of the process should not lead to any changes in the hierarchical structure of cities. 
significantly greater than one. This means that size diverges towards its means, namely, the larger a city, the larger the expected growth. Then it is straightforward that the variance ratio, $\theta$, increases for both large and small cities. However, it should be noted that the variance of small cities, $\theta$, is always greater than the variance of large cities and that the variance of growth $\sigma_{B I G}^{2}$ is always less than $\sigma^{2}$ small; thus, although we cannot formally show evidence of generalised Gibrat's law (in particular regarding statement b)), we again show a greater volatility for small cities when $q>1$.

The analysis carried out over these three countries provides an important first conclusion. On the basis of statement b), we have shown that when $q>1$ and Gibrat's law holds $(\beta=1)$, the variance $\left(\theta\right.$ - and $\sigma^{2}$ parameters) is actually greater for small cities. Moreover, when the $q$-coefficient is greater than one but decreasing, we have modifications on the growth process of large cities, but not on those of small cities: in particular, the larger the city, the lower the expected growth. On the other hand, when the $q$ coefficient is greater than one, but increasing, small cities present the opposite growth process: namely the larger the city, the larger the expected growth. Consequently, we find evidence of the generalised Gibrat's' law - as in statement b) of Section 1 - in the countries displaying $q>1$, where the independence of the mean with respect to the size is in operation, while the same is not true for the variance.

A reasonable criticism, at this point in the analysis, could be that generally, small entities (cities, firms and so on) present a greater volatility than larger ones. We can anticipate that we will find the opposite evidence in the case of $q<1$ in the next Sub-section.

\subsection{Luxembourg}

Luxembourg shows an estimated $q$-coefficient lower than one. It increases until 1930, and after that it is not significantly different from one. Between 1821-1922, we are in condition c) of Section 1. Considering the entire sample, we have already shown that Gibrat's law holds in the first three years of the sample (1851-1880) and in 1922 with estimated $\beta$-parameters not significantly different from one. Moreover, considering the two sub-samples, most of the time we find evidence of Gibrat's law for both large and small cities. At a first glance it seems that large and small cities face the same underlying growth processes. However, to test statements c), we need to take into account the behaviour of the variance.

The variance ratio for the entire sample in Luxembourg in the period 1821-1922 is always greater than one, indicating a greater volatility of the process as time goes by. Indeed, when we split the sample in 
two halves, the variance ratios for the large cities show values always greater than one, while, for the small cities, they are always below one. This implies an (increasing) change in the underlying volatility of the growth process for large cities, in contrast to a (decreasing) change in the underlying volatility of the growth process for small cities. Moreover, in those years, the variance of growth $\sigma_{B I G}^{2}$ is always

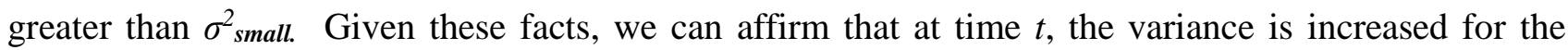
large cities but decreased for the small cities, indicating a dependence of variance with respect to size; in particular, smaller cities face a lower volatility than large cities. In summary, statement c), which affirms: "if $q<1$, In order that Gibrat's law occurs, it is necessary that the mean is independent from the city size but not the variance, indeed the associate growth process requires that smaller cities face a lower volatility of growth than larger cities", is satisfied for the whole sample in 1821-1930.

In the period 1935-2011, the $q$-coefficient is not statistically different from one. By considering the relationship with Gibrat's law, we then investigate condition a) of Section 1 which predicts that the associated growth process requires that smaller cities face the same growth as larger cities. Considering the entire sample, we have already shown that Gibrat's law holds most of the time (estimated $\beta$ parameters not significantly different from one). Considering the two sub-samples, we find similar evidence for both large and small cities, even with same exceptions. However it is interesting to note that in those years where Gibrat's law does not hold, the estimated parameters $\beta, \theta$ and $\sigma^{2}$ show very different behaviour (i.e. $\theta_{B I G}=.965$ and $\theta_{\text {small }}=1.162 ; \sigma_{B I G}^{2}=1.93$ and $\sigma_{\text {small }}^{2}=1.31$ in 1970), but, in general, in those years where Gibrat's law holds, the differences between the estimators are not so large (i.e. $\theta_{B I G}=1.03$ and $\theta_{\text {small }}=1.01$ and $\sigma_{B I G}^{2}=0.55$ and $\sigma_{\text {small }}^{2}=0.53$ in 1947). In summary, statement a) which affirms: "if $q=1$, then in order that Gibrat's law occurs neither the mean nor the variance of growth can depend on size" is satisfied for the whole sample.

\subsection{Synthesis}

A synthesis of the above results - confirming the hypotheses by Cordoba (2003) - is presented in Table 6.

\section{< Table 6. About Here >}

The analysis carried out in this section prompts several interesting conclusions. We have been able to empirically verify the presence of a "generalised Gibrat law", as theoretically predicted by Cordoba (2003). In particular, we have verified statements a), b) and c) of Section 1. In more detail, we have shown that when $q>1$ (statement b)) and Gibrat's law holds $(\beta=1)$, the variance-ratio $(\theta$-parameter) and 
the variance of growth, $\sigma^{2}$, are actually higher for the small cities, in comparison to that for large cities, indicating a larger volatility for small cities. On the contrary, when $q<1$ (statement c)) and Gibrat's law holds $(\beta=1)$, the variance-ratio ( $\theta$-parameter) and the variance of growth, $\sigma^{2}$, is actually lower for the small cities, in comparison to that for large cities, indicating a larger volatility for the smaller ones. When $q=1$ (statement a)) and Gibrat's law holds, our findings agree with previous research, as both the mean and variance appear to be independent from the size.

Moreover, when the $q$-coefficient is greater than one but decreasing, we have modifications on the growth process of large cities, but not on those of small cities; in particular, the larger the city, the lower the expected growth. On the other hand, when $q>1$ but increasing, small cities present the opposite growth process, namely the larger the city, the larger the expected growth. We have, of course, an opposite behaviour when the $q$-coefficient is less than one.

\section{Conclusion}

The aim of our research work was to explore specific conditions leading to a generalisation of Gibrat's law in connection with the different typologies of rank-size distribution. For this purpose we empirically explored the link between the rank-size exponent, $q$, with the necessary conditions for Gibrat's law (that is mean and variance of the growth have to be independent from the size). We started our analysis based on the conclusion of Cordoba (2003, p. 3): "Pareto distributions with larger exponents (more unequal distributions) require more volatile growth processes". As far as we know, the conventional methodologies (Section 3) used to test Gibrat's law do not address this issue. In particular, a greater (lower) volatility of the variance is usually not empirically envisaged. We showed, instead, that, according to Cordoba (2003), the variance can be dependent on size if the rank-size coefficient is different from one; in particular, we verified what Cordoba (2003) calls a "generalised Gibrat's law" for different countries with different spatial-economic characteristics: Botswana, Germany, Hungary and Luxembourg. We found strong evidence of this generalised Gibrat's law for Botswana and Luxembourg. We found weak evidence of Gibrat's law for Germany and no evidence for Hungary.

Our results confirm the propositions provided by Cordoba (2003). In particular, when $q=1$, neither the mean nor the variance of growth depend on size; when $q>1$, the mean is independent of the city size, but not the variance, and small cities face a greater volatility in growth than larger cities; alternatively, when $q<1$, the mean is independent from the city size, but not the variance, and large cities face a greater volatility in growth than smaller ones. Gibrat and Zipf have offered complementary perspectives on city size and systems of cites in a given country. Their contributions are not necessarily 
identical, but offer new perspectives on the same multi-faceted prism of the space-economy. These results might be useful to 'relax' Gibrat's law in its strict interpretation, by reinforcing the hypothesis that small entities face a greater volatility in the growth process.

Our analysis prompts various intriguing research questions in the future. While Gibrat's law and Zipf's law mirror important organised structures in the topology of systems of cities, other relevant structural patterns may be investigated as well, such as the existence of fractal structures in urban systems (based, for example, on Mandelbrot's principles) or the persistent existence of spatial population or socioeconomic disparities (based, for example, on Herfindahl's index). Clearly, the dynamics of such processes deserve due attention. In addition, the above applied investigation also calls for more fundamental research into the functional or behavioural backgrounds of such regularities. Three research directions are important here; (a) the interdependence between population indicators and broader socio-economic indicators for a system of cities; (b) the degree of various cities in the same national system; (c) the relationship between recent strong evolutionary trends in the digital world and the development of cities (and systems of cities). 


\section{References}

Adamic, L. A., 2000. Zipf, power-laws, and Pareto - a ranking tutorial, retrieved from www.hpl.hp.com Auerbach, F., 1913. Das Gesetz Der Bevolkerungs Konzentration, Petermanns Geographische Mitteilungen. $59,73-76$.

Auerbach, F., 1915. The law of population concentration. Scientific American Supplement. 76, 384.

Black, D., Henderson V., 2003. Urban evolution in the USA. Journal of Economic Geography. 3(4), 343372.

Bottazzi, G., Dosi, G., Lippi, M., Pammolli, F., Riccaboni, M., 2001. Innovation and corporate growth in the evolution of the drug industry. International Journal of Industrial Organization. 19(7), 1161-1187.

Champernowne, D. G., 1953. A model of income distribution. The Economic Journal. 63(250), 318-351.

Chesher, A., 1979. Testing the law of proportionate effect. Journal of Industrial Economics. 27, 403-411.

Christaller, W., 1933. Die Zentrale Orte in Süddeutschland. Eine Ökonomisch-Geografische Untersuchung

Über Die Gesatzmässigkeit Der Verbreitung Und Entwicklung Der Siedlungen Mit Städtischen Funktionen, Gustav Fischer, Jena.

Cordoba, J., 2003. On the distribution of city sizes. Urban/Regional. 0302002, EconWPA.

Cordoba, J., 2008. A generalized Gibrat's law. International Economic Review. 49(4), 1463-1468.

Cuberes, D., 2011. Sequential city growth: empirical evidence. Joournal of Urban Economics. 69(2), 229239.

Eeckhout, J., 2004. Gibrat's law for (all) cities. American Economic Review. 94(5), 1429-1451.

Fazio, G., Modica, M., 2012. Pareto or log-normal? A recursive truncation approach to the distribution of (all) cities. Working Papers 2012_10, Business School, Economics, University of Glasgow, Glasgow.

Gabaix, X., 1999. Zipf's law for cities: an explanation. The Quarterly Journal of Economics. 114(3), 739767.

Gabaix, X., Ibragimov, R., 2011. Rank-1/2: a simple way to improve the OLS estimation of tail exponents. Journal of Business \& Economic Statistics. 29(1), 24-39.

Gabaix, X., Ioannides, Y. M., 2004. The evolution of city size distributions, in J.V. Henderson and J.-F. Thisse (eds), Handbook of Regional and Urban Economics, Vol. 4. Elsevier, Amsterdam, 2341-2378.

Garmestani, A.J., Allen, C.R., Gallagher, C.M., Mittelstaedt, J.D., 2007. Departures from Gibrat's law, discontinuities and city size distributions. Urban Studies. 44(10), 1997-2007.

Gibrat, R., 1931. Les Inégalités Economiques, Libraire du Recueil Siray, Paris.

Giesen, K., Suedekum, J., 2011. Zipf's law for cities in the regions and the country. Journal of Economic Geography. 11(4), 667-686. 
González-Val, R., 2010. The evolution of U.S. city size distribution from a long-term perspective (19002000). Journal of Regional Science. 50(5), 952-972.

González-Val, R., Lanaspa, L., Sanz, F., 2012. New evidence on Gibrat's law for cities. Working Papers 2012/18. Institut de Economia de Barcelona (IBE), Barcelona.

Guerin-Pace F., 1995. Rank-size distribution and the process of urban growth. Urban Studies. 32(3), 551562.

Henderson, J.V., Wang, H.G., 2007. Urbanization and city growth: the role of institutions. Regional Science and Urban Economics. 37, 283-313.

Ioannides, Y. M., Overman, H. G., 2003. Zipf's law for cities: an empirical examination. Regional Science and Urban Economics. 33(2), 127-137.

Kalecki, M., 1945. On the Gibrat distribution. Econometrica. 13(2), 161-170.

Lösch, A., 1940. The Economics of location. Yale University Press, New Haven (translated by W. Woglam and W. Stolper in 1954).

Paelinck, J.H.P., Nijkamp, P., 1976. Operational Theory and Method in Regional Economics, Saxon House, Farnborough.

Parr, J. B., 1985. A note on the size distribution of cities over time. Journal of Urban Economics. 18, 199212.

Rosen, K. T., Resnick, M., 1980. The size distribution of cities: An examination of the Pareto law and primacy. Journal of Urban Economics. 8(2), 165 -186.

Simon, H., 1955. On a class of skew distribution functions. Biometrika. 42(3/4), 425-440.

Singer, H. W., 1930. The "Courbe Des Populations". A parallel to Pareto's law. The Economic Journal. 48(182), 254-263.

Soo, K. T., 2005. Zipf's law for cities: a cross-country investigation. Regional Science and Urban Economics. 35(3), 239-263.

Soo, K. T., 2007. Zipf's Law and Urban Growth in Malaysia. Urban Studies. 44(1), 1-14

Steindl, J., 1968. Size distributions in Economics, in International Encyclopedia of the Social Sciences, Vol. 14, Silks, ed. (New York, Macmillan and the Free Press).

Zipf, G., 1949. Human behavior and the principle of least effort: an introduction to human ecology, Addison-Wesley, Cambridge. 
Table 1. Rationale for countries selection

\begin{tabular}{|c|c|c|c|c|c|}
\hline & & \multicolumn{2}{|c|}{ Population Density } & \multicolumn{2}{|c|}{ GDP p.c } \\
\hline & & High & Low & High & Low \\
\hline \multirow{2}{*}{$\stackrel{\mathbb{N}}{\text { is }}$} & High & Germany & Botswana & Germany & Botswana \\
\hline & Low & Luxembourg & Hungary & Luxembourg & Hungary \\
\hline
\end{tabular}

Table 2. Spatial Economic Characteristics of the Five Countries under Analysis

\begin{tabular}{|c|c|c|c|c|c|c|c|c|c|c|}
\hline Country & Year & $\begin{array}{c}\mathrm{Km}^{2} \\
\text { (thousands) }\end{array}$ & $\begin{array}{c}\text { Pop. } \\
\text { (milion) }\end{array}$ & Density & \% Urban pop. & $\begin{array}{l}\text { \% pop. } \\
\text { growth }\end{array}$ & $\begin{array}{l}\text { Railway } \\
* 1000 \text { km }\end{array}$ & $\begin{array}{l}\text { Roadway } \\
* 1000 \text { km }\end{array}$ & GDP p. c. & Growth (\%) \\
\hline Botswana & 2011 & 581 & 1.85 & 3.19 & $62.00 \%$ & 1.47 & 0.90 & 25.80 & 9,481 & $5.1 \%$ \\
\hline Germany & 2007 & 357 & 81.78 & 229 & $74.00 \%$ & -0.20 & 41.90 & 644.50 & 40,403 & $2.7 \%$ \\
\hline Hungary & 2011 & 93 & 9.99 & 107.4 & $69.00 \%$ & -0.18 & 8.10 & 197.50 & 13,045 & $1.70 \%$ \\
\hline Luxembourg & 2011 & 2,5 & 0.51 & 205.6 & $85.00 \%$ & 1.13 & 0.27 & 5.20 & 106,958 & $1.00 \%$ \\
\hline
\end{tabular}

Table 3. Descriptive Statistics of the Five Countries under Analysis

\begin{tabular}{cccccccc}
\hline Country & Year & N. cities & $\ln ($ Mean) & $\ln$ (Variance) & $\ln$ (Median) & Skewness & Kurtosis \\
\hline Botswana & 2011 & 461 & 7.15 & 1.22 & 6.97 & 0.78 & 2.23 \\
Germany & 2007 & 12,262 & 7.42 & 1.50 & 7.30 & 0.34 & 0.17 \\
Hungary & 2011 & 3,154 & 6.75 & 1.34 & 6.70 & 0.40 & 0.95 \\
Luxemburg & 2011 & 116 & 7.81 & 0.92 & 7.61 & 0.86 & 1.45 \\
\hline
\end{tabular}


Table 4a. Model A Estimates (Countries: Botswana, Germany, Hungary and Luxembourg; Different Years)

\begin{tabular}{|c|c|c|c|c|c|c|}
\hline Country & Year & $\beta$ & Robust s.e. & $\theta$ & $\mathbf{R}^{2}$ & N. obs. \\
\hline Botswana & 2011 & $.995 * *$ & .0149 & 1.0782 & .92 & 460 \\
\hline \multirow[t]{14}{*}{ Germany } & 1994 & .999 & .0003 & 1.0009 & .99 & 12,280 \\
\hline & 1995 & $.999^{*}$ & .0002 & .9983 & .99 & 12,291 \\
\hline & 1996 & $.999 *$ & .0003 & .9987 & .99 & 12,291 \\
\hline & 1997 & .998 & .0002 & .9978 & .99 & 12,291 \\
\hline & 1998 & .998 & .0002 & .9984 & .99 & 12,291 \\
\hline & 1999 & $1.00 * * *$ & .0003 & 1.0011 & .99 & 12,293 \\
\hline & 2000 & $.999 * * *$ & .0003 & .9998 & .99 & 12,294 \\
\hline & 2001 & 1.001 & .0001 & 1.0024 & .99 & 12,294 \\
\hline & 2002 & 1.001 & .0001 & 1.0017 & .99 & 12,294 \\
\hline & 2003 & $1.00^{*}$ & .0001 & 1.0009 & .99 & 12,293 \\
\hline & 2004 & 1.001 & .0001 & 1.0027 & .99 & 12,292 \\
\hline & 2005 & 1.001 & .0001 & 1.0031 & .99 & 12,293 \\
\hline & 2006 & 1.001 & .0001 & 1.0027 & .99 & 12,993 \\
\hline & 2007 & 1.001 & .0002 & 1.0039 & .99 & 12,259 \\
\hline \multirow[t]{3}{*}{ Hungary } & 1990 & 1.055 & .0019 & 1.1215 & .99 & 3,121 \\
\hline & 2001 & 1.034 & .0022 & 1.0806 & .99 & 3,121 \\
\hline & 2011 & 1.028 & .0025 & 1.0674 & .99 & 3,121 \\
\hline \multirow[t]{17}{*}{ Luxembourg } & 1851 & $.941^{*}$ & .0264 & .9557 & .93 & 116 \\
\hline & 1871 & $.993^{* *}$ & .0276 & 1.0779 & .92 & 116 \\
\hline & 1880 & $1.005 * *$ & .0306 & 1.0667 & .95 & 116 \\
\hline & 1890 & 1.048 & .0183 & 1.0855 & .93 & 116 \\
\hline & 1900 & 1.109 & .0324 & 1.2833 & .96 & 116 \\
\hline & 1910 & 1.079 & .0187 & 1.1803 & .99 & 116 \\
\hline & 1922 & $1.01 * *$ & .0123 & 1.0473 & .97 & 116 \\
\hline & 1930 & 1.103 & .019 & 1.2406 & .98 & 116 \\
\hline & 1935 & $1.001 * * *$ & .007 & 1.0059 & .99 & 116 \\
\hline & 1947 & $1.014 *$ & .0061 & 1.0355 & .99 & 116 \\
\hline & 1960 & 1.069 & .0132 & 1.1725 & .98 & 116 \\
\hline & 1970 & 1.044 & .0156 & 1.1198 & .97 & 116 \\
\hline & 1981 & $1.016^{* *}$ & .0144 & 1.0576 & .98 & 116 \\
\hline & 1991 & $.993 * *$ & .0104 & .9964 & .99 & 116 \\
\hline & 2001 & 0.955 & .0076 & .9199 & .99 & 116 \\
\hline & 2002 & 0.994 & .0015 & .989 & .99 & 116 \\
\hline & 2003 & $.995^{* *}$ & .0034 & .9926 & .99 & 116 \\
\hline
\end{tabular}

$*$ Significant at $1 \% * *$ significant at $5 \% * * *$ significant at $10 \%$ 
Table 4b. Model A Estimates (Country: Luxembourg; Different Years)

\begin{tabular}{|c|c|c|c|c|c|c|}
\hline Country & Year & $\beta$ & Robust s.e. & $\theta$ & $\mathbf{R}^{2}$ & N. obs. \\
\hline \multirow[t]{8}{*}{ Luxembourg } & 2004 & $.997 * *$ & .0018 & .9947 & .99 & 116 \\
\hline & 2005 & $.996 * *$ & .0024 & .9926 & .99 & 116 \\
\hline & 2006 & $.997 * *$ & .0015 & .9961 & .99 & 116 \\
\hline & 2007 & 0.992 & .0029 & .9851 & .99 & 116 \\
\hline & 2008 & 0.992 & .0023 & .985 & .99 & 116 \\
\hline & 2009 & $.996 * *$ & .0019 & .994 & .99 & 116 \\
\hline & 2010 & $.996^{*}$ & .0016 & .9924 & .99 & 116 \\
\hline & 2011 & $.999 * *$ & .0015 & 1.00 & .99 & 116 \\
\hline
\end{tabular}

*** Significant at $1 \% * *$ significant at $5 \% *$ significant at $10 \%$ 
Table 5a. The Zipf's and Gibrat's Parameters (Countries: Botswana, Germany, Hungary and Luxembourg; Different Years)

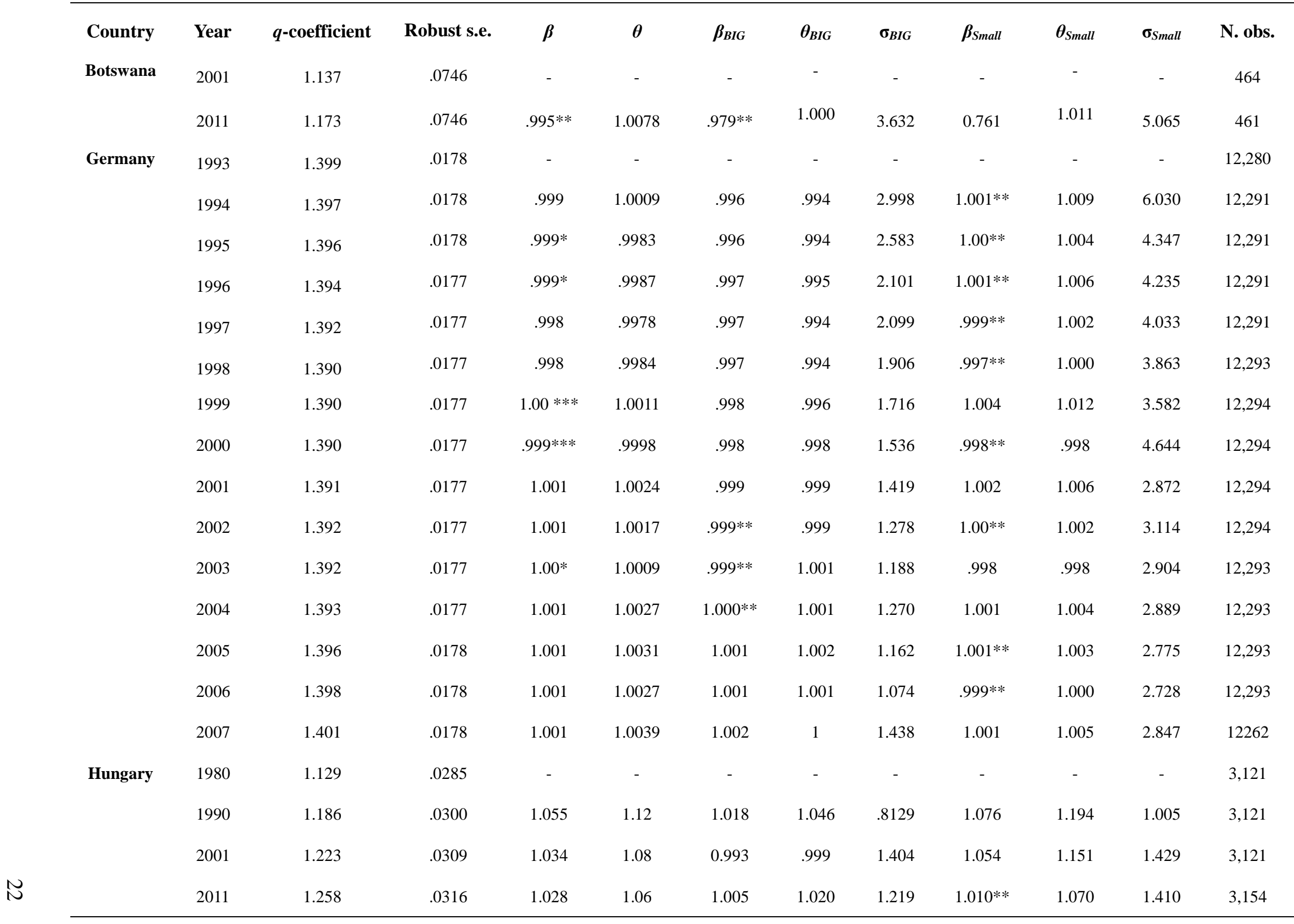

$*$ Significant at $1 \% * *$ significant at $5 \% * * *$ significant at $10 \%$ 
Table 5b. The Zipf's and Gibrat's Parameters (Countries: Botswana, Germany, Hungary and Luxembourg; Different Years)

\begin{tabular}{|c|c|c|c|c|c|c|c|c|c|c|c|c|}
\hline Country & Year & $q$-coefficient & Robust s.e. & $\beta$ & $\theta$ & $\beta_{B I G}$ & $\theta_{B I G}$ & $\sigma_{B I G}$ & $\boldsymbol{\beta}_{\text {Small }}$ & $\theta_{\text {Small }}$ & $\sigma_{\text {Small }}$ & N. obs. \\
\hline \multirow[t]{26}{*}{ Luxembourg } & 1821 & .5031 & .0660 & - & - & - & - & - & - & - & - & 116 \\
\hline & 1851 & .4965 & .0652 & $.941^{*}$ & .9557 & $.949^{* *}$ & .978 & .6141 & .730 & .764 & .7822 & 116 \\
\hline & 1871 & .5154 & .0676 & $.993 * *$ & 1.0779 & $.962 * *$ & 1.070 & 1.296 & $.887 * *$ & .981 & .6157 & 116 \\
\hline & 1880 & .5350 & .0702 & $1.005 * *$ & 1.0667 & $1.003 * *$ & 1.060 & 1.693 & $.811^{* *}$ & .871 & 1.904 & 116 \\
\hline & 1890 & .5881 & .0772 & 1.048 & 1.0855 & $.995 * *$ & 1.160 & 3.327 & $.944 * *$ & .965 & .6451 & 116 \\
\hline & 1900 & .6744 & .0885 & 1.109 & 1.2833 & 1.115 & 1.340 & 2.651 & $.947 * *$ & .969 & .6763 & 116 \\
\hline & 1910 & .7350 & .0965 & 1.079 & 1.1803 & 1.091 & 1.210 & 1.332 & $.986 * *$ & 1.035 & 6178 & 116 \\
\hline & 1922 & .7500 & .0984 & $1.01 * *$ & 1.0473 & $0.998^{* *}$ & 1.025 & 2.095 & $1.042 * *$ & 1.140 & .5105 & 116 \\
\hline & 1930 & $.8377^{* *}$ & .1100 & 1.103 & 1.2406 & 1.090 & 1.240 & 2.574 & $1.01^{* *}$ & 1.048 & .5981 & 116 \\
\hline & 1935 & $.8391 * *$ & .1101 & $1.001 * * *$ & 1.0059 & $.986^{* *}$ & .976 & 1.045 & $1.033^{*}$ & 1.081 & .7215 & 116 \\
\hline & 1947 & $.8543^{* *}$ & .1121 & $1.014^{*}$ & 1.0355 & $1.013^{* *}$ & 1.034 & .5543 & $.982 * *$ & 1.009 & 5250 & 116 \\
\hline & 1960 & $.9252 * *$ & .1214 & 1.069 & 1.1725 & $1.011^{* *}$ & 1.059 & 1.395 & $1.006^{* *}$ & 1.109 & .7906 & 116 \\
\hline & 1970 & $.9735^{* *}$ & .1278 & 1.044 & 1.1198 & 0.965 & .963 & 1.930 & $1.016^{* *}$ & 1.162 & 1.308 & 116 \\
\hline & 1980 & $.9923 * *$ & .1302 & $1.016^{* *}$ & 1.0576 & 0.942 & .922 & 1.815 & $1.022 * *$ & 1.150 & 1.268 & 116 \\
\hline & 1991 & $.9803 * *$ & .1287 & $.993 * *$ & .9964 & 0.949 & .920 & 1.395 & $1.053^{* *}$ & 1.145 & .881 & 116 \\
\hline & 2001 & $.9409 * *$ & .1235 & 0.955 & .9199 & 0.949 & .907 & .874 & .910 & .871 & 1.337 & 116 \\
\hline & 2002 & $.9365^{*}$ & .1229 & 0.994 & .989 & $.998 * *$ & .997 & 1.291 & $.990^{* *}$ & .983 & 2.177 & 116 \\
\hline & 2003 & $.9330^{* *}$ & .1225 & $.995 * *$ & .9926 & $1.00^{* *}$ & 1.00 & 1.366 & $.997 * *$ & 1.00 & 3.356 & 116 \\
\hline & 2004 & $.9302 * *$ & .1221 & $.997 * *$ & .9947 & $1.00 * *$ & 1.00 & 1.209 & $1.00^{* *}$ & 1.020 & 2.465 & 116 \\
\hline & 2005 & $.9270 * *$ & .1217 & $.996^{* *}$ & .9926 & $.995^{* *}$ & .992 & 1.529 & $.992 * *$ & .989 & 2.812 & 116 \\
\hline & 2006 & $.9253 * *$ & .1215 & $.997 * *$ & .9961 & 1.001 & 1.00 & 1.109 & $.994 * *$ & .991 & 2.254 & 116 \\
\hline & 2007 & $.9195 * *$ & .1207 & 0.992 & .9851 & 0.994 & .988 & 1.412 & $.974^{* *}$ & .955 & 3.498 & 116 \\
\hline & 2008 & $.9140 * *$ & .1200 & 0.992 & .985 & $.999 * *$ & .998 & 1.291 & .976 & .955 & 2.501 & 116 \\
\hline & 2009 & $.9120 * *$ & .1197 & $.996 * *$ & .994 & $1.00 * *$ & 1.00 & 1.672 & $.989 * *$ & .981 & 2.219 & 116 \\
\hline & 2010 & $.9094 * *$ & .1194 & $.996^{*}$ & .9924 & $1.00^{* *}$ & 1.00 & 1.247 & $.986^{*}$ & .974 & 1.787 & 116 \\
\hline & 2011 & $.9094 * *$ & .1194 & $.999 * *$ & 1.00 & $1.00^{* *}$ & 1.00 & 1.073 & $.997 * *$ & .996 & 2.137 & 116 \\
\hline
\end{tabular}


Table 6. Relationship between Zipf/Rank-Size and Generalized Gibrat

\begin{tabular}{|c|c|c|c|c|c|c|c|}
\hline Country & Year & $q$ & $q$ trend & Gibrat & $\theta$ & $\sigma^{2}$ & $\begin{array}{c}\text { Generalized Gibrat } \\
\text { validity }\end{array}$ \\
\hline Botswana & 2001-2011 & $>1$ & $\uparrow$ & TRUE $(\beta=1)$ & $\theta_{B I G}<\theta_{\text {small }}$ & $\sigma_{B I G}^{2}<\sigma_{\text {small }}^{2}$ & $\begin{array}{c}\text { YES } \\
\text { (statement b)) }\end{array}$ \\
\hline \multirow{2}{*}{ Germany } & 1993-1999 & $>1$ & $\downarrow$ & $\operatorname{WEAK}^{13}(\beta=1)$ & $\theta_{B I G}<\theta_{\text {small }}$ & $\sigma_{B I G}^{2}<\sigma_{\text {small }}^{2}$ & $\begin{array}{c}\text { YES } \\
\text { (statement b)) }\end{array}$ \\
\hline & $2000-2007$ & $>1$ & $\uparrow$ & $\operatorname{FALSE}(\beta \neq 1)$ & $\theta_{B I G}<\theta_{\text {small }}$ & $\sigma_{B I G}^{2}<\sigma_{\text {small }}^{2}$ & NO \\
\hline Hungary & 1980-2011 & $>1$ & $\uparrow$ & $\operatorname{FALSE}(\beta \neq 1)$ & $\theta_{B I G}<\theta_{\text {small }}$ & $\sigma_{B I G}^{2}<\sigma_{\text {small }}^{2}$ & NO \\
\hline \multirow{2}{*}{ Luxembourg } & $1821-1930$ & $<1$ & $\uparrow$ & $\operatorname{TRUE}(\beta=1)$ & $\theta_{B I G}>\theta_{\text {small }}$ & $\sigma_{B I G}^{2}>\sigma_{\text {small }}^{2}$ & $\begin{array}{c}\text { YES } \\
\text { (statement c)) }\end{array}$ \\
\hline & 1935-2011 & $=1$ & $\leftrightarrow$ & $\operatorname{TRUE}(\beta=1)$ & $\theta_{B I G}=\theta_{\text {small }}$ & $\sigma_{B I G}^{2}=\sigma_{\text {small }}^{2}$ & $\begin{array}{c}\text { YES } \\
\text { (statement a)) }\end{array}$ \\
\hline
\end{tabular}

13 Notice that weak Gibrat's law means that Gibrat's law holds only for few years. 


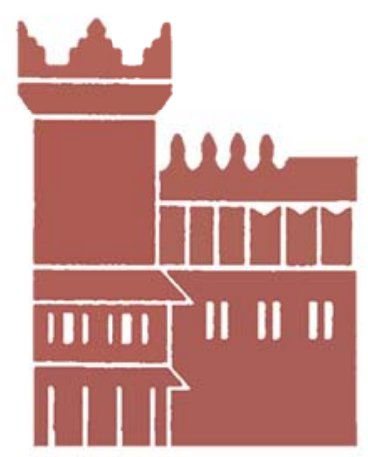

Alma Mater Studiorum - Università di Bologna DEPARTMENT OF ECONOMICS

Strada Maggiore 45

40125 Bologna - Italy

Tel. +39051 2092604

Fax +390512092664

http://www.dse.unibo.it 